\title{
A fs-frequency comb referenced diode laser system for coherent spectroscopy of cold molecules
}

\author{
A. Wicht ${ }^{1}$, N. Strau $\mathbf{B}^{1}$, K. Döringshoff ${ }^{1}$, I. Ernsting ${ }^{1}$, B. Roth ${ }^{1}$, \\ J. Koelemeij ${ }^{1}$, S. Schiller ${ }^{1}$, R.-H. Rinkleff ${ }^{2}$, K. Danzmann ${ }^{2}$ \\ ${ }^{\prime}$ Institut für Experimentalphysik, Heinrich-Heine-Universität Düsseldorf, Germany \\ ${ }^{2}$ Institut für Gravitationsphysik, Leibniz-Universität Hannover, Germany \\ andreas.wicht@uni-duesseldorf.de
}

\begin{abstract}
A new type of diode laser system for precision spectroscopy is presented. Its excellent passive stability eases locking to fs-frequency combs, which is demonstrated with high resolution spectroscopy of cold $\mathrm{HD}^{+}$ions.
\end{abstract}

The advent of fs-frequency comb based optical synthesizers not only revolutionizes optical metrology but also provides the means to perform coherent spectroscopy of cold (mK range) molecular ensembles which requires the generation of multi-chromatic optical fields with ultra-stable difference frequencies in the THz range. We aim at the generation of molecular coherence between different individual rotational states of the electronic and vibrational ground state of cold molecular $\mathrm{HD}^{+}$ions. The multi-chromatic radiation fields are generated by diode lasers which have to be phase-locked to a Ti:Sa based fs-frequency comb. Phase-locking of diode lasers to a frequency comb is challenging because of the relatively low signal-to-noise ratio of the corresponding RF beat notes and the relatively large phase noise bandwidth of grating diode lasers.

We present the second generation of a diode laser system which is based on merging the concepts underlying the grating diode laser and the diode laser with resonant optical feedback (GEECDL, Grating Enhanced External Cavity Diode Laser). The diode laser system is designed for precision coherent spectroscopy of cold $\mathrm{HD}^{+}$ions between $1380 \mathrm{~nm}$ and $1500 \mathrm{~nm}$. The first diffraction order of a volume holographic transmission grating is coupled into a folded confocal cavity. Resonant light from that cavity is fed back onto the laser diode. The zero order output of the grating is used as the laser output. This way we combine the good over-all and continuous tuneability and the simple operation of grating lasers with the narrow line width provided by diode lasers with resonant optical feedback. With a first demonstrator operating at $850 \mathrm{~nm}$ [1] we have proven the feasibility of the concept: an overall tuneability of $40 \mathrm{~nm}$ has been achieved, a continuous tuneability of $40 \mathrm{GHz}$ was demonstrated, and the free running short term linewidth was determined to be below $50 \mathrm{kHz}$.

We report on the over-all and continuous tuneability and the passive frequency stability of the second generation laser system. To demonstrate its capability for precision spectroscopy we frequency-lock the laser to an $\mathrm{H}$-maser referenced Ti:Sa based fs-frequency comb and analyze the relative line width and frequency stability. As an additional demonstration of the laser system performance we report on fs-frequency comb based high resolution spectroscopy of cold $\mathrm{HD}^{+}$molecular ions. The absolute accuracy is currently limited by properties of the molecular ensemble and corresponds to better than $1 \mathrm{MHz}$. The absolute accuracy of the laser system frequency is on the order of $10 \mathrm{kHz}$ in this measurement.

We are currently preparing the third generation laser system. It features a different laser diode chip and a modified diode laser setup, in which the feedback optics is located on one side of the diode chip and the laser output is emitted on the opposite side [2]. This way higher laser output power and stable operation also at higher operating currents is expected. With this new setup we expect to increase the laser output power from about $1 \mathrm{~mW}$ to $50 \mathrm{~mW}$. With this new laser we will also be able to determine the short term laser line width provided by this laser concept in a beat note measurement with the second generation laser system. We report on first results of phase locking both lasers to each other.

This work is supported by the Deutsche Forschungsgemeinschaft. JK acknowledges support by the Alexander von Humboldt-Foundation.

[1] A. Wicht, P. Huke, R.-H. Rinkleff, K. Danzmann, „Advancing the optical feedback concept: grating enhanced external cavity diode laser", Physica Scripta T118, 82 (2005)

[2] We gratefully acknowledge technological support by Sacher Lasertechnik, Marburg, Germany 